\title{
Broadband Spectral Analysis of Non-Debye Dielectric Relaxation in Percolating Heterostructures
}

\author{
E. Tuncer ${ }^{1}$, J. Belattar 2 , M. E. Achour ${ }^{2}$ and C. Brosseau ${ }^{3}$ \\ ${ }^{1}$ Applied Superconductivity Group, Fusion Energy Division, \\ Oak Ridge National Laboratory, Oak Ridge, TN 37831-6122, \\ ${ }^{2}$ Laboratoire des Systèmes de Télécommunications et d'Ingénierie de la Décision, \\ Département de Physique, Faculté des Sciences, \\ Université Ibn Tofail, B. P. 133, 14000 Kénitra, \\ ${ }^{3}$ Université Européenne de Bretagne, Université de Brest, Lab-STICC and Département de \\ Physique, CS 93837, 6 avenue Le Gorgeu, 29238 Brest Cedex 3, \\ 1 USA \\ ${ }^{2}$ Morocco \\ ${ }^{3}$ France
}

\section{Introduction}

Dielectric relaxation in complex materials has been fascinating physicist for more than half a century ${ }^{1-5}$. Much of the present understanding of dynamics in a very wide range of complex dielectric systems (e.g. filled polymers, glasses, biopolymers, liquid crystals, and even concrete) currently revolves around the ideas of spatial and dynamic heterogeneity. These complex materials in general exhibit nontrivial relaxation dynamics on both microscopic and macroscopic scales. Fundamental questions remain not only about dynamics, but also about how randomness and connectedness give rise to the dielectric attributes. Addressing such questions is quite challenging because the structural aspects of random composites are still largely unknown. ${ }^{1-7}$ Within this perspective, a number of experiments, e.g. dielectric spectroscopy experiments ${ }^{8-9}$ and computer simulations, e.g. Monte Carlo and finite element calculations $6,7,10-13$ have progressed in parallel. Models for dielectric relaxation in heterostructures can be broadly placed in two groups.

The first picture has been heavily investigated in various phenomenological models of dielectric relaxation. Previous results suggest that pure Debye response is virtually nonexistent in complex solids. As an alternative to the Debye model, investigators have used the purely empirical analytical expressions, one of them is called Cole-Cole ${ }^{14}$ and it explores the spectral dependence of (relative) complex permittivity $\varepsilon$ as a function of angular frequency $\omega, \varepsilon=\varepsilon_{\infty}+\left(\varepsilon_{s}-\varepsilon_{\infty}\right) \frac{1}{1+(i \omega \tau)^{1-\alpha}}$, where $\varepsilon_{s}$ and $\varepsilon_{\infty}^{\prime}$ are the static $\left(\omega<<\tau^{-1}\right)$ and the high frequency $\left(\omega>>\tau^{-1}\right)$ permittivity values for a given relaxation, which can be considered atomic, electronic, ionic or interfacial polarization. In addition, it is assumed that the non- 
negative empirical exponent $a$ gauges the broadening of the dielectric loss spectrum. Jonscher ${ }^{15}$ suggested an alternate expression for the imaginary part of the permittivity $\varepsilon^{\prime \prime}$, $\varepsilon^{\prime \prime}=\frac{\varepsilon^{\prime \prime}{ }_{m}}{(\omega \tau)^{-m}+(\omega \tau)^{1-n}}$, where the exponents $m$ and $n$ lie between zero and unity. They determine the shape of the response at frequencies lower and higher than the maximum frequency $\omega_{\max }$ at which the maximum value of $\varepsilon^{\prime \prime}$ is observed $\left[\varepsilon^{\prime \prime}{ }_{m}=\max \left(\varepsilon^{\prime \prime}\right)\right]$, i.e. the loss peak. The physical process that gives rise to this peak is a dipolar re-orientation. Several studies of these spectral models have been carried out to analyze the fractional exponents $m$ and $n$ of the power-law decays. This behavior has been observed in carbon black (CB)-epoxy composites frequency over the range from $200 \mathrm{~Hz}$ to $15 \mathrm{MHz},{ }^{16}$ and other types of binary (conductor-insulator) composites containing nanoparticles. ${ }^{17}$ We point out that permanent and induced dipoles, electron conduction in CB aggregates, Maxwell-Wagner-Sillars (MWS) interfacial polarization all contribute to the effective (collective) relaxation behavior of the composite material. Based upon Jonscher's framework, we assume that the medium consists of a cluster of fluctuating reorientable dipoles and/or small size polar entities. Jonscher's model relies on the notion that in a typical solid the microscopic dipolar, electronic or ionic transitions are very rapid (on the scale of ps or less) and they take place in a system in which electrostatic or strain interactions are invariably present but the adjustment of local equilibrium takes a longer time to be realized. ${ }^{15}$ In other words, the cluster structure of a dipolar system may be considered as a natural consequence of the fact that when the electric field is on, only some of the dipoles have enough energy and time to reach a configuration state aligned along the electric field. Thus, the dielectric response originates with specific, spatially limited regions containing dipoles with positions altered by the applied electric field and their random environment. During the relaxation process the strongly coupled local (intracluster) motions are expected to be generated first, and then followed by the weakly coupled (intercluster) motions, which produce the long-range structure. ${ }^{15}$

An alternative approach to the above empirical expressions is the so-called continuous distribution of relaxation times 18-19 The distribution of relaxation times reveals the true relaxation spectrum of a system. The complex effective permittivity $\varepsilon(\omega)$ is defined for an ensemble of Debye processes with a continuous relaxation time distribution, $\varepsilon(\omega)=\varepsilon_{\infty}+\left(\varepsilon_{s}-\varepsilon_{\infty}^{\prime}\right) \int_{-\infty}^{\infty} \frac{g[\ln (\tau)]}{1+i \omega \tau} d[\ln (\tau)]$, where $g(\tau)$ is the distribution of relaxation times. Each relaxation has its own relaxation strength $\left(\varepsilon_{s}-\varepsilon_{\infty}^{\prime}\right)$, and it is therefore natural to keep relaxation time and strength of each Debye process together in a numerical approach, then $\int_{-\infty}^{\infty} g[\ln (\tau)] d[\ln (\tau)]=\varepsilon_{s}-\varepsilon_{\infty}^{\prime}$. Although, the concept of distribution of relaxation times can be notoriously misleading by inducing numerical instabilities, and in certain cases lead to ambiguities in interpretation of dielectric data ${ }^{18}$, linearization of the problem and using the Monte Carlo integration hypothesis puzzle out the instability and ill-conditioned nature of the problem. ${ }^{19}$ Numerous examples of distribution of relaxation times are reported in different systems by one of the present authors (ET). ${ }^{20}$ The distribution $g(\tau)$ constructed captures correctly the relaxation properties. One should note immediately that, except for some special cases, e.g. Cole-Cole, inverting these integrals is nontrivial since they are nonlinear. ${ }^{8}$

It should be emphasized that a direct comparison between these different approaches has been rarely attempted except in Tuncer and collaborators. ${ }^{19-20}$ The major focus of this work 
is to provide such a comparison based on experimental data on CB filled epoxy composites, thereby establishing the bridge between these two widely used complementary theoretical approaches to broadband relaxation in dielectric materials.

Experiments were conducted with CB particles (Raven 2000, Raven 5000, Raven 7000 obtained from the Columbian Chemicals Company USA and Monarch 1100 purchased from Cabot Company USA) and diglycidylic ether of bisphenol F (DGEBF) epoxy (Araldite XPY 306, epoxy equivalent weight=172) obtained from Ciba Geigy Ltd. The mixture of DGEBF and $\mathrm{CB}$ particles was processed with an amine curing agent (4,9-dioxadodecan-1,12diamine, equivalent weight $=81$, supplied by BASF, and used without further purification). Resin was cured at room temperature for $24 \mathrm{~h}$. The neat DGEBF has a dc conductivity of 1.4 10-14 $\Omega^{-1} \mathrm{~m}^{-1}$, a density of $1.19 \mathrm{gcm}^{-3}$, and a glass transition temperature at. $T_{\mathrm{g}}=83{ }^{\circ} \mathrm{C}$. The different series of samples were fabricated by mechanical mixing. ${ }^{16}$ The $\mathrm{CB}$ volume fraction within the composite is denoted as $\phi_{2}$. The values of the percolation threshold, $\phi_{c}$, for DGEBF/Raven 2000, DGEBF/Raven 5000, DGEBF/Raven 7000, and DGEBF/Monarch 1100 were determined by dc conductivity measurements (linear current-voltage relationship) and are equal to $3.6,10,2.75$ and $8 \mathrm{vol} \%$, respectively. The effective (relative) complex permittivity of the composite samples was measured in the frequency $(F=\omega / 2 \pi)$ range from $200 \mathrm{~Hz}$ to $15 \mathrm{MHz}$. using a HP 4194A impedance analyzer. The experimental setup and procedures for measuring $\varepsilon(F, T)$, where the temperature $T$ ranges from ambient temperature up to $70{ }^{\circ} \mathrm{C}$, are similar to those previously described.16 Note that the contribution of the Ohmic conductivity, i.e. $\sigma_{\mathrm{dc}}\left(\phi_{2}, T\right) / \varepsilon_{0} \omega$ was removed to the raw $\varepsilon$ " data.

Room temperature isotherms of complex permittivity in Cole-Cole representation are shown in Fig. 1 for several CB concentrations of the DGEBF/Raven 2000 series. Superimposed on these experimental data is a nonlinear least-squares fit to the Cole-Cole form with fitting parameters $\varepsilon_{\mathrm{s}}, \varepsilon_{\infty}, \alpha$, and $\tau$. Evolution of the best-fit parameters with CB concentration is presented in Fig. 2. As concentration $\phi_{2}$ increases from $\approx 0$ to close to the percolation threshold, $\varepsilon_{\mathrm{s}}$ (resp. $\varepsilon_{\infty}^{\prime}$ ) increases by $300 \%$ (resp. $75 \%$ ). The estimated exponent $\alpha$ deviates significantly from 1 . For this range of $\mathrm{CB}$ volume fraction $\phi_{2}$ extends over nearly 2 orders of magnitude.

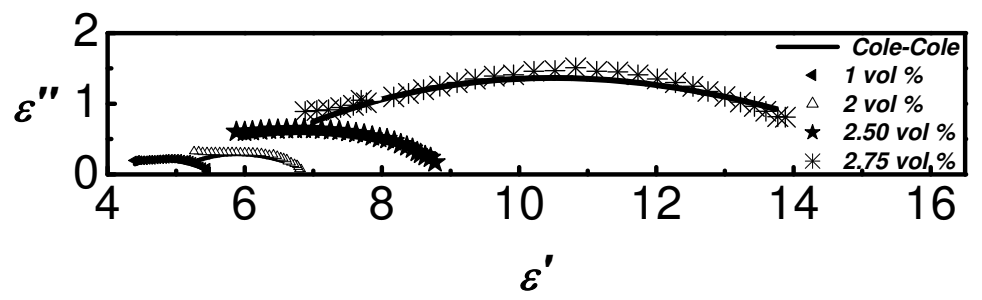

Fig. 1. Cole-Cole plot $\varepsilon^{\prime \prime}$ as a function of $\varepsilon^{\prime}$ for concentration $\phi_{2}=0.1,0.5,1$, and 2 vol $\%$ (Raven 2000-DGEBF samples). Room temperature. Symbols are experimental data and solid curves are 4-parameter fit to the Cole-Cole equation. The numbers in the graph indicate the value of the $\mathrm{CB}$ loading 


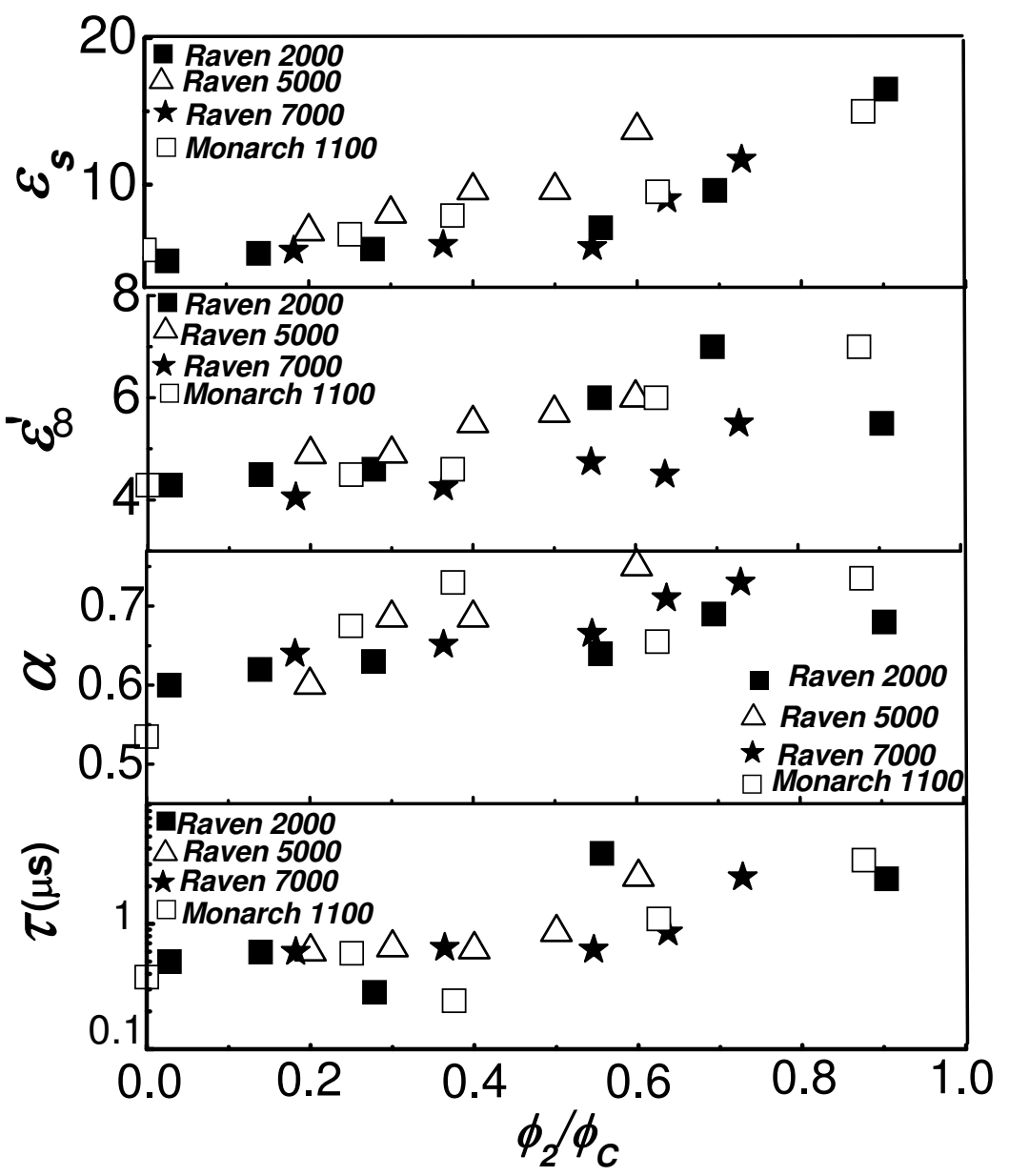

Fig. 2. (a) Plot of the static (relative) permittivity $\varepsilon_{s}$ vs $\phi_{2} / \phi_{c}$ for the different series of samples. The symbols in the graph indicate the type of CB particles. (b) Same as in (a) for the limiting permittivity at high frequencies $\varepsilon_{\infty}^{\prime}$. (c) Same as in (a) for the Cole-Cole exponent $\alpha$. (d) Similar to (a) for the relaxation time $\tau$ at room temperature

For $T \leq T_{g}$ the temperature dependence of $\tau$ typically follows the Vogel-Tammam-Fulcher (VTF) thermal activation law for the slowing down of relaxation processes found in many amorphous materials. ${ }^{16,19}$ As shown in Fig. 3, with increasing temperature $T$, the $\tau$ isotherms exhibit decreasing evolution. Assuming that the temperature dependence of $\tau$ between $20^{\circ} \mathrm{C}$ and $70^{\circ} \mathrm{C}$ is of the form $\tau=\bar{\tau}_{0} \exp \left[W / k\left(T-T_{0}\right)\right]$, the best fit of our data using maximum likelihood method yields $\bar{\tau}_{0} \approx 10^{-8} \mathrm{~s}, 267 \leq T_{0} \leq 292{ }^{\circ} \mathrm{C}$ which is an ordering temperature lower than $T_{\mathrm{g}}$ whose significance has remained unclear (there is no microscopic 
prescription to calculate $T_{0}$ ), and apparent free energy of thermal activation $W=0.63 \pm 0.19$ $\mathrm{meV}$, which we interpret as the average potential barrier between different sites inside the heterostructure. To put numbers in context, we note that the values for $W$ reported in the literature for similar materials range are consistent with ours. ${ }^{12-13,18}$ The value of $\bar{\tau}_{0}$ is almost 3 orders of magnitude larger than $\bar{\tau}_{0} \approx 10^{-11} \mathrm{~s}$, commonly used for the preexponential factor. A possible interpretation of this pre-exponential factor is to assume that a dipole on average visits $N=\bar{\tau}_{0} / \tau_{0} \approx 10^{3}$ sites inside the cluster before being relaxed. Similar behavior, i.e. long trapping time, has been reported for bulk silicate filled carboxylated nitrile rubber. ${ }^{21-22}$ Meanwhile, the values of $\alpha$ remain $\approx 0.70 \pm 0.05$ in this range of $T$.

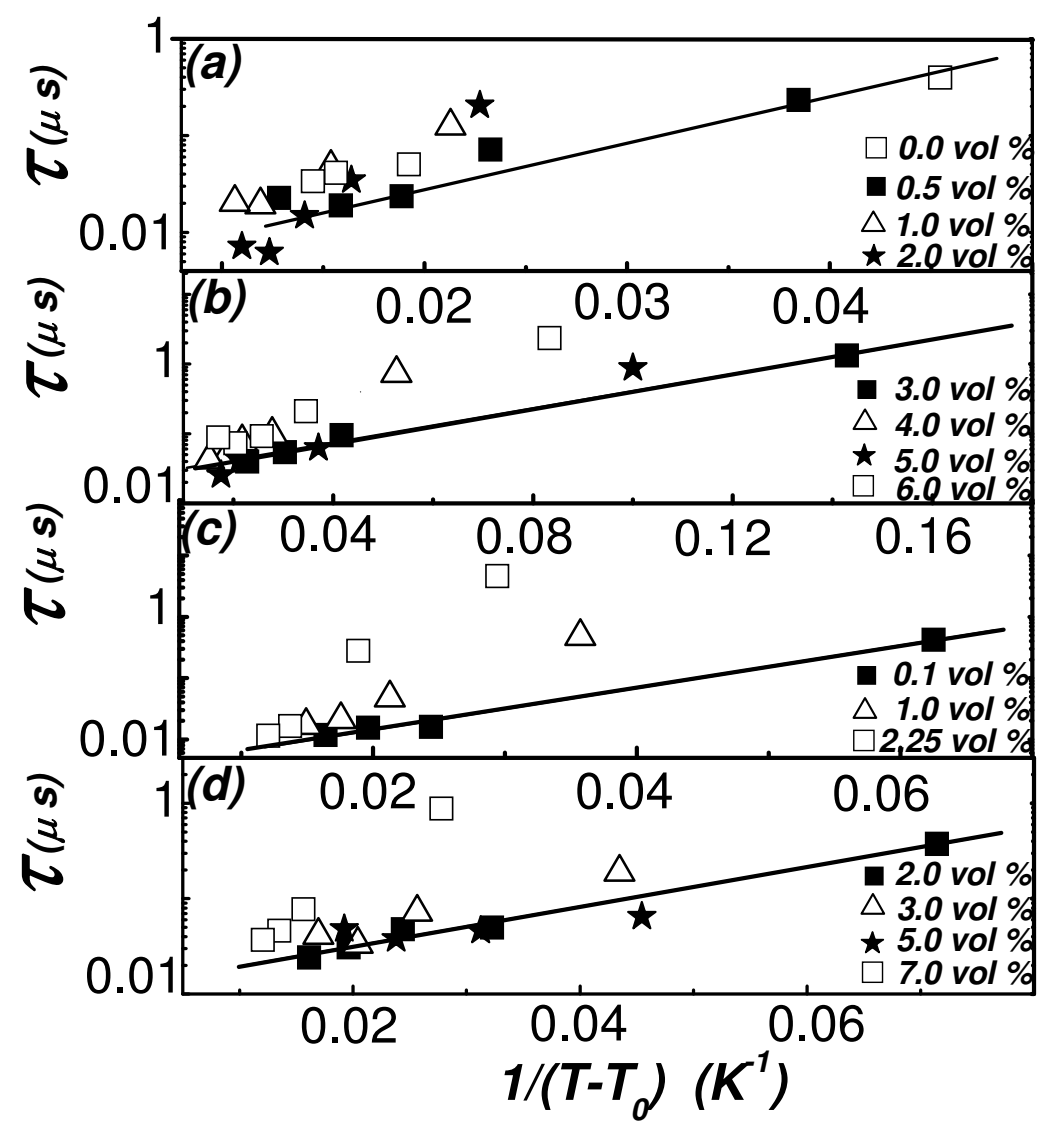

Fig. 3. (a) Plot of $\tau$ as a function of temperature for Raven 2000-DGEBF samples and different values of CB volume fractions. The solid curve is a fit to the Vogel-TammamFulcher law to the data represented by solid squares (see text). The numbers in the graph indicate the value of $\phi_{2}$. (b) Same as in (a) for Raven 5000-DGEBF samples. (c) Same as in (a) for Raven 7000-DGEBF samples. (d) Same as in (a) for Monarch 1100-DGEBF samples 


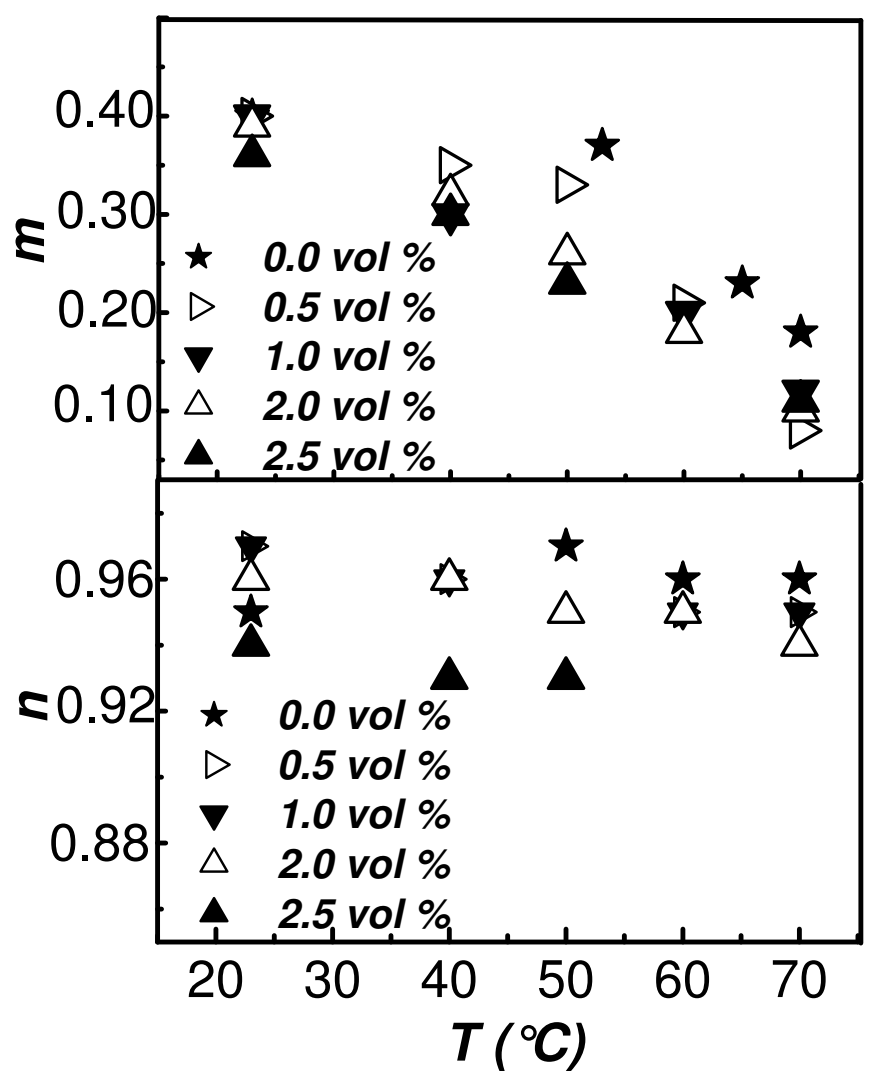

Fig. 4. (a) Plot of $m$ vs $T$ for the different concentrations of Raven 2000 in the Raven 2000 DGEBF samples. The symbols in the graph indicate the value of the CB loading. (b) Same as in (a) for $n$

So far, we have only discussed the Cole-Cole phenomenology. In order to correlate the temperature dependence of the relaxation mechanisms for the samples investigated here and better understand how temperature affects the values of $n$ and $m$ obtained from our variable-temperature measurements are graphed in Fig. 12 for the Raven 2000-DGEBF as an illustrative example. A similar behavior is observed for the other series of samples. Two typical behaviors corresponding to the temperature range explored are: $m$ decreases strongly with $T$ and $n$ is close but smaller than 1 . Such comparison can provide a comprehensive picture of the similarities and differences between the $\mathrm{CB}$ types in the overall relaxation behavior of these filled polymers. By contrast, our results are less clear for the $m$ dependence as a function of CB content because the fits yield larger uncertainty for $m$. Jonscher suggested to associate the values of $m$ and $n$ with the degree of correlation between the flip-flop transitions (fast intra-cluster interactions) for $m$ and between the flip transitions (slow inter-cluster interactions) for $n$, respectively..$^{15}$ According to this modeling, flip transitions represent tunnelling modes between different configurations giving a net change of the total dipole moment and flip-flop transitions represent local fluctuations of the dipole 
moment. The latter retains the average value of the total polarization and may be regarded as synchronous transitions in opposite sense at different points in the material, giving zero net change of dipole moment. The value $m=0$ corresponds to completely uncorrelated flipflop transitions of the system. The limit $n=0$, corresponds to a complete screening, as in a free charge system, while $n=1$ corresponds to the absence of screening, as would be the case with immobile charges (Debye system) which are unable to follow local changes of potential. ${ }^{13}$ In contrast to the classical Debye response, corresponding to a system of noninteracting permanent dipoles, ${ }^{13}$ the dielectric response arises as being due to a highly dispersive and lossy system yielding a frequency independent $\varepsilon^{\prime \prime} / \varepsilon^{\prime}$ ratio.

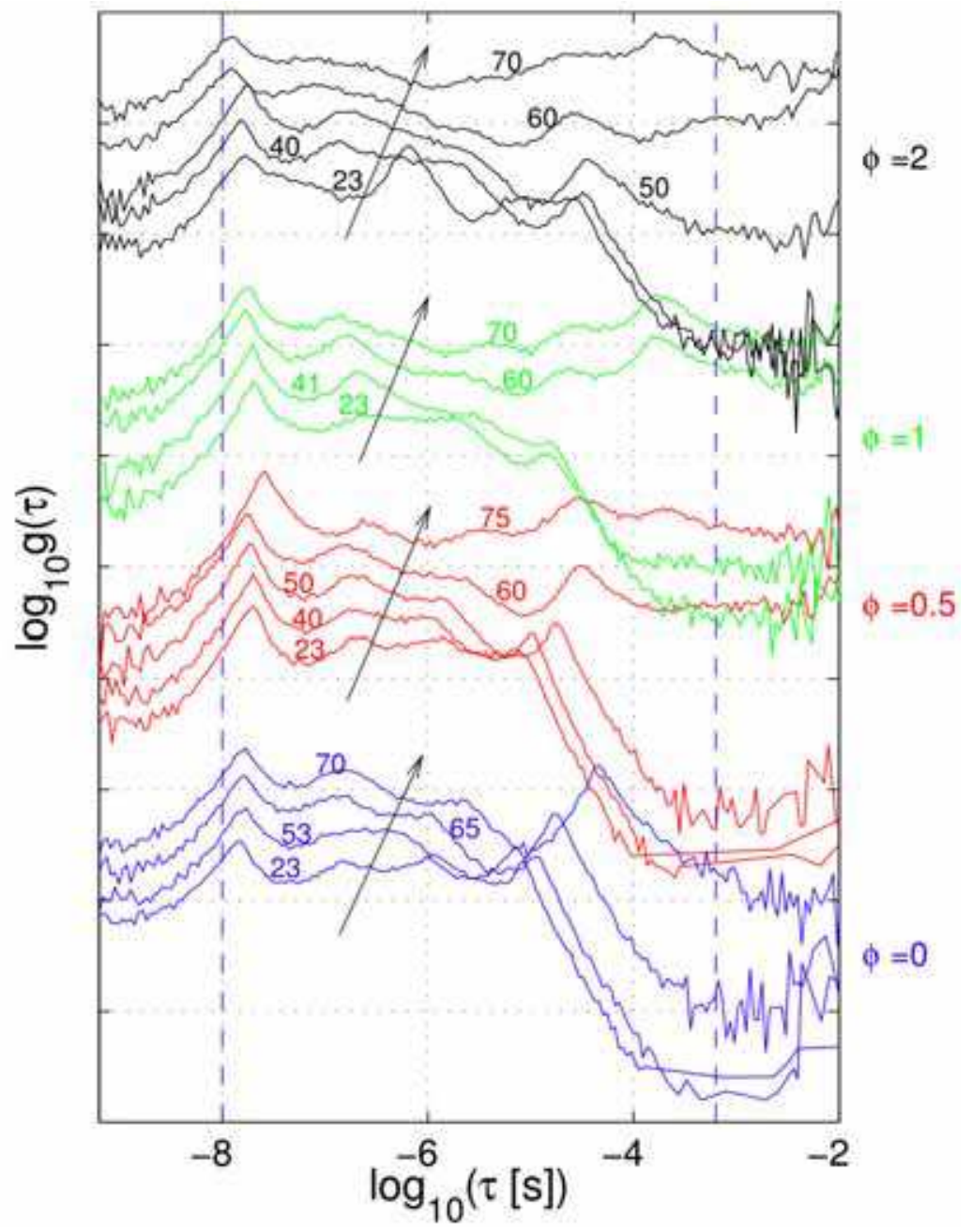

Fig. 5. (Color online) The relaxation time distributions $g(\tau)$ for all samples of Raven 2000DGEBF. The numbers on each curve indicate the temperature. The volume fractions are shown on the right side of the graph. The distributions are shifted to unravel the relaxations 
The principle of the numerical analysis of a set of experimental frequency-response data that involves a continuous distribution of relaxation times is identical to that described elsewhere. ${ }^{19-20}$ Fig. 5 shows the results of this analysis and shows a comparison of $g(\tau)$ at different temperatures and CB content for Raven 2000-DGEBF samples. The data are shifted to guide the eyes, and each relaxation spectrum had similar baseline, which is shown in Fig 6. The vertical dashed lines in Fig. 5 are drawn to indicate the measurement window for the reciprocal frequency. The relaxations resolved at fast times had clear peaks due to coupling of the high frequency permittivity with high frequency relaxations - the relaxations were not fully observed within the current experimental frequency window. Even KramersKronig transformation ${ }^{23}$ would resolve an accurate relative permittivity when a relaxation is not observed fully. The relaxations resolved were broad and distributed over the relaxation time axis. The low temperature data for all the samples $\left(23^{\circ} \mathrm{C}\right.$ and $\left.40^{\circ} \mathrm{C}\right)$ had the long relaxation times observable within the experimental frequency window - this is shown in Fig. 5 with a sharp drop in the distribution after $10^{-5-10-4} \mathrm{~s}$. This characteristic is most visible in the unfilled sample $\left(\phi_{2}=0\right)$ over all the temperatures, where in other words the polarizations were finalized. Over these temperatures the distributions of the filled samples exhibited continuation of the polarization in the materials. The reason for the change in the relaxation spectra with temperature in filled samples was due to the influence of the $C B$ particles on the energy landscape - the local field and the dynamics of polymer motion were altered. One can also speculate about the ionic species and their dynamics at long times with addition of fillers. Currently there is none systematic investigations on the change in relaxation times spectra in filled systems.
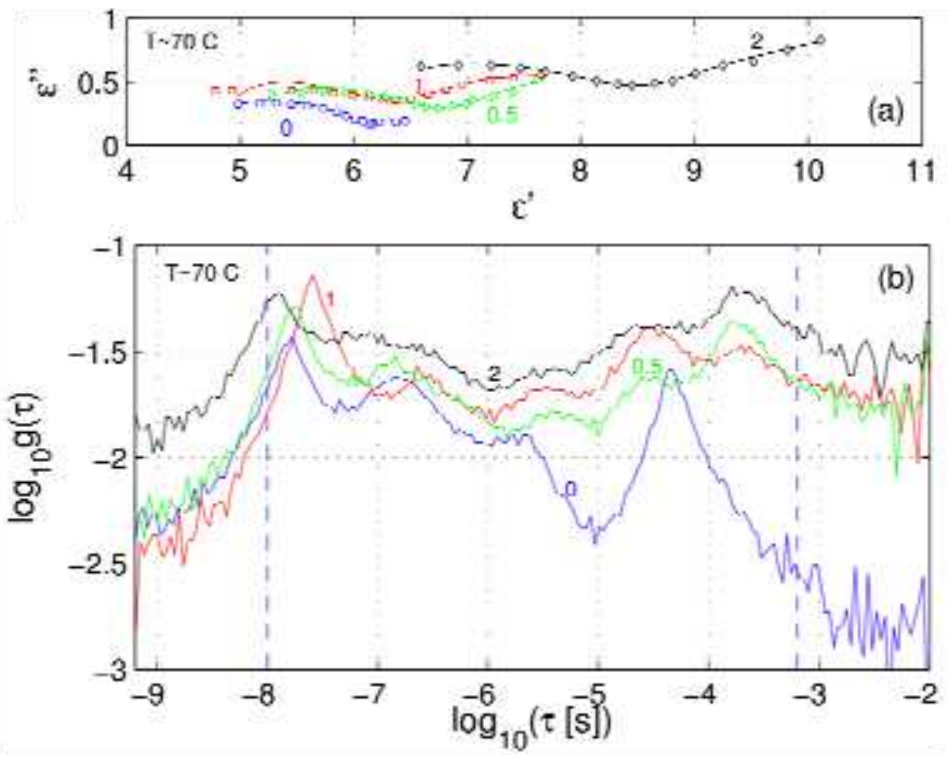

Fig. 6. (Color online) (a) Cole-Cole plot, and (b) resolved distributions for samples with CB volume percentage $0,0.5,1$ and 2 . The complex permittivities that are close to the glass transition are shown. The dashed lines in (b) indicate the reciprocal experimental frequency range that probes to the relaxation times 
One striking observation of the analysis method is presented in Fig. 6 for different volume fraction of $\mathrm{CB}$ at high temperatures $\left(>65^{\circ} \mathrm{C}\right)$. The relaxations resolved around medium times $\left(10^{-7} \mathrm{~s}\right.$ and $\left.10^{-5} \mathrm{~s}\right)$ could be followed through all samples from unfilled to $2 \mathrm{vol} \%$. Similar kind of response was previously observed and reported by Tuncer et al. ${ }^{24}$ This sort of behaviour should be expected since the signature of the matrix material should be detectable with the impedance measurements and the applied analysis, unless the matrix physically changed with the addition of fillers. To illustrate how the resolved distributions were actually describing the data, the dielectric response generated from the distribution data are plotted on the experimental values with solid lines in Fig. 6. There is very good agreement with measurements and the distribution of relaxation times analysis. Finally, we show the relaxation spectra and dielectric data estimated for composite samples using room temperature data in Fig. 7. The evolution of the relaxation spectra with filler content is noteworthy. As shown with examples, the distribution of relaxation times approach is a compelling tool to better understand composite materials.

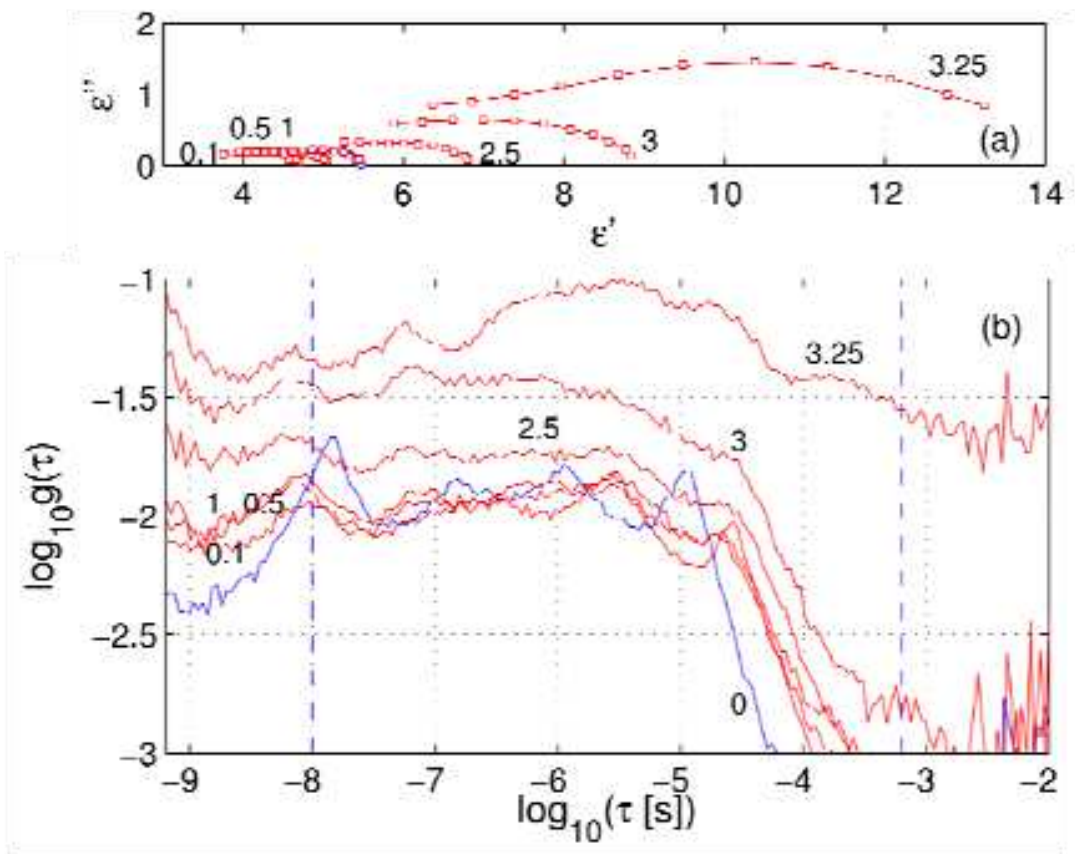

Fig. 7. (Color online) (a) Cole-Cole plots, and (b) resolved distributions for samples with different $\mathrm{CB}$ volume percentages. The data are obtained at room temperature, and concentrations of the samples are below the percolation threshold. The dashed lines in (b) indicate the reciprocal experimental frequency range that probes to the relaxation times

In summary, a combination of several complimentary analytical techniques has been used to investigate the dielectric relaxation in carbon black-epoxy composites. The results presented here confirm the non-Debye relaxation behavior in these percolative heterostructures that was identified by the analysis of the permittivity spectra of these heterostructures which can be accounted for by the Cole-Cole phenomenological equation. The relaxation time follows a 
VTF temperature dependence with activation energy of the order of the meV. Comparison can be also made with an alternative explanation based on the concept of a set of dipoles making sudden jumps between fixed orientations in space and causing a slow screening response in the surrounding medium. ${ }^{13}$ This deviation from Debye's behavior is assumed to be due to many-body effects on relaxation processes described by two fractional exponents $m$ and $n$ characterizing the power-law decays of this spectral model. The exponents $m$ and $n$ are both positive and smaller than unity. Interestingly, they have different temperature variations: while $m$ is strongly decreasing with increasing temperature $\left(<T_{\mathrm{g}}\right), n$ takes a value close to 1 . Our results suggest that many body interactions have a dominant role to explain the collective coupling between the dipole ensemble and the environment. However accurate they may be, each modeling method considered in this work has one important approximation which is difficult to overcome: effective medium approximation, i.e. when spatial inhomogeneities manifest on scales much smaller than the relevant length scale in the system, an average homogeneous treatment is justified. A continuous distribution of relaxation times has been obtained from experimental data following the method of one of the authors (ET). Our results raise the question of how the parameters of empirical expressions $\alpha, m$ and $n$ can be interpreted. Unlike these empirical model parameters the $g(\tau)$ obtained from distribution of relaxation times is not based on a model, the relaxation spectrum is a true representation of the material - the spectrum does not change with choice of initial parameters or does not require a-priori assumption. A detailed physical (microscopic) interpretation of these quantities depends on both of the cluster structure and the collective nature of the interactions. Yet the problem is so important that numerical answers that include multi-fractal geometry are highly desirable. It would be very attractive to perform spectral density analysis on the composite response ${ }^{25}$ to separate structural information from the intrinsic properties of the constituents. In that case the structure whether it is fractal or not would be resolved with the analysis, however, in that case one needs to have the full information on the electrical properties of the constituents.

We end by making a number of comments about some standing questions and future directions. Various questions can still be addressed. Interaction with interfaces is one of the main relaxation mechanisms for dipoles. In order to deal with interfaces it will be interesting to consider relaxation spectra based on $a b$ initio Monte Carlo and molecular dynamics simulations. Since most properties of random composites are determined by topology, the beginning of any such study is the creation of experimentally credible structural models. Studies in this direction would benefit from basic information on the mesostructure obtained via atomic force microscopy. ${ }^{26}$ A fully microscopic theory of relaxation in percolative heterostructures is outstanding and remains an exciting prospect. One should employ the spectral density representation to completely realize the electrical properties of composite materials.

\section{Acknowledgments}

M. E. A. thanks the Universite de Brest for its financial support and hospitality where some of this work was completed. This work was supported by the Lab-STICC, Unite Mixte de Recherche CNRS 3192. E.T. was sponsored by the U.S. DOE Office of Electricity Delivery and Energy Reliability--Advanced Cables and Conductors, under contract DE-AC05-00OR22725 with Oak Ridge National Laboratory, managed and operated by UT-Battelle, LLC. 


\section{References}

[1] R. Landauer, in Electrical Transport and Optical Properties of Inhomogeneous Media, edited by J. C. Garland and D. B. Tanner (American Institute of Physics, New York, 1978), vol. 40, pp. 2-43.

[2] A. H. Sihvola, Electromagnetic Mixing Formulas and Applications (IEE Publishing, London, 1999).

[3] D. J. Bergman and D. Stroud, in Solid State Physics, Advances in Research and Applications, edited by H. Ehrenreich and D. Turnbull (Academic, New York, 1992), Vol. 46.

[4] S. Torquato, Random Heterogeneous Materials: Microstructure and Macroscopic Properties (Springer, New York, 2002).

[5] M. Sahimi, Heterogeneous Materials I: Linear Transport and Optical Properties (Springer, New York, 2003).

[6] C. Brosseau, J. Phys. D 39, 1277 (2006); C. Brosseau and A. Beroual, Prog. Mater. Sci. 48, 373 (2003).

[7] E. Tuncer and S. M. Gubanski, J. Appl. Phys. 8, 310 (2001).

[8] H. Schäfer, E. Sternin, R. Stannarius, M. Arndt, and F. Kremer, Phys. Rev. Lett. 76, 2117 (1996); F. Kremers and A. Schönhals, eds., Broadband Dielectric Spectroscopy (Springer, Berlin, 2003), and J. R. Macdonald, ed., Impedance Spectroscopy (Wiley, New York, 1987); A. Bello, E. Laredo, and M. Grimau, Phys. Rev. B 60, 12764 (1999).

[9] Y. Feldman, A. Andrianov, E. Polygalov, G. Romanychev, I. Ermolina, Y. Zuev, and B. Milgotin, Rev. Sci. Instrum. 67, 3208 (1996); Y. Feldman, A. Puzenko, and Y. Ryabov, Chem. Phys. 284, 139 (2002).

[10] E. Tuncer and S. M. Gubanski, in Dielectric and Related Phenomena DRP'98: Polymers and Liquid Crystals, edited by A. Wlochowicz and E. Targosz-Wrona, Technical University of Lodz, Branch in Bielsko-Biala, Poland (SPIE, Bellingham, WA, 1998), pp. 136-142; E. Tuncer, Licenciate thesis-Tech. Rep. 338 L, Chalmers University of Technology, Gothenburg, Sweden, 2000; E. Tuncer, Dielectric relaxation in dielectric mixtures, PhD thesis, Chalmers University of Technology, Gothenburg, Sweden, 2001; E. Tuncer, S. M. Gubanski, and B. Nettelblad, J. Appl. Phys. 89, 8092 (2001); E. Tuncer, B. Nettelblad and S. M. Gubanski, J. Appl. Phys. 92, 4612 (2002); E. Tuncer, S. M. Gubanski, and B. Nettelblad, J. Electrostatics 56, 449 (2002); E. Tuncer, Turk. J. Phys. 27, 121 (2003); E. Tuncer and E. Tuncer, Turk. J. Phys. 23, 101 (2003); E. Tuncer. Phys. D: Appl. Phys. 37, 334 (2004); R. F. Hamou, J. R. Macdonald and E. Tuncer, J. Phys.: Condens. Matter 21, 025904 (2009).

[11] V. Myroshnychenko and C. Brosseau, Phys. Rev. E 71, 016701 (2005); V. Myroshnychenko and C. Brosseau, J. Appl. Phys. 97, 044101 (2005); V. Myroshnychenko and C. Brosseau, J. Phys. D 41, 095401 (2008); V. Myroshnychenko and C. Brosseau, J. Appl. Phys. 103, 084112 (2008); V. Myroshnychenko and C. Brosseau, IEEE Trans. Dielectr. EI 16, 1209 (2009). See also A. Mejdoubi and C. Brosseau, Phys. Rev. B 74, 165424 (2006); A. Mejdoubi and C. Brosseau, Phys. Rev. E 74, 031405 (2006); A. Mejdoubi and C. Brosseau, Phys. Rev. B 74, 165424 (2006); A. Mejdoubi and C. Brosseau, J. Appl. Phys. 100, 094103 (2006); A. Mejdoubi and C. Brosseau, J. Appl. Phys. 101, 084109 (2007).

[12] J. P. Calame, A. Birman, Y. Carmel, D. Gershon, B. Levush, A. A. Sorokin, V. E. Semenov, D. Dadon, L. P. Martin, and M. Rosen, J. Appl. Phys. 80, 3992 (1996); J. P. Calame, J. Appl. Phys. 94, 5945 (2003). 
[13] L. C. Shen, C. Liu, J. Korringa, and K. J. Dunn, J. Appl. Phys. 67, 7071 (1990); C. Liu and L. C. Shen, Modelling and Simulation in Materials Science and Engineering, 1, 723 (1993); P. K. Ghosh and M. E. Azimi, IEEE Trans. Dielec. Insul. 1, 975 (1994).

[14] B. K. P. Scaife, Principles of Dielectrics (Clarendon Press, Oxford, 1998); K. S. Cole and R. H. Cole, J. Chem Phys. 9 (1941) 341.

[15] A. K. Jonscher, Dielectric Relaxation in Solids, (Chelsea Dielectric Press, London, 1987). See also A. K. Jonscher, Universal Relaxation Law, (Chelsea Dielectric Press, London, 1996) and more recently A. K. Jonscher, J. Phys. D 32, R57, (1999). See also L. A. Dissado and R. M. Hill, J. Chem. Soc. Farady Trans. 2, 291 (1984).

[16] M. E. Achour, C. Brosseau, and F. Carmona, J. Appl. Phys. 103, 094103 (2008); C. Brosseau and M. E. Achour, J. Appl. Phys. 105, 124102 (2009).

[17] J. Wu and D. S. McLachlan, Phys. Rev. B 58, 14880 (1998); D. S. McLachlan, W. D. Heiss, C. Chiteme, and J. Wu, Phys. Rev. B 58, 13558 (1998); J. Wu and D. S. McLachlan, Phys. Rev. B 56, 1236 (1997); ibidem 58, 14880 (1998).

[18] J. R. Mcdonald, J. Appl. Phys. 62, R51 (1987).

[19] E. Tuncer and S. M. Gubanski IEEE Trans. Dielectr. EI, 8, 310 (2001).

[20] E. Tuncer, M. Furlani, B.-E. Mellander, J. Appl. Phys. 95, 3131 (2004); E. Tuncer, M. Wegener, R. Gerhard-Multhaupt, J. Non-Cryst. Solids 351, 2917 (2005); E. Tuncer, M. Wegener, P. Frubing, and R. Gerhard-Multhaupt, J. Chem. Phys. 122, 084901 (2005); E. Tuncer, J.R. Macdonald, J. Appl. Phys. 99, 074106 (2006); J. R. Macdonald and E. Tuncer, J. Electroanalytical Chem. 602, 255 (2007); E. Tuncer, G. Polizos, I. Sauers, D. R. James, A. R. Ellis, J. M. Messman, T. Aytug Cryogenics 49, 463 (2009).

[21] N. G. McCrum, B. E. Read, and G. Williams, Anelastic and Dielectric Effects in Polymeric Solids (Dover, New York, 1991); G. Strobl, The Physics of Polymers (Springer, Berlin, 1996), and C. A. Angell, in Relaxations in Complex Systems 1, edited by K. Ngai and G. B. Wright, (Natl. Technol. Inform. Ser. U.S. Dept. of Commerce, Springfield, VA, 1985).

[22] J. Fritzsche, A. Das, R. Jurk, K. W. Stöckelhuber, G. Heinrich, and M. Klüppel, eXPRESS Polym. Lett. 2, 373 (2008).

[23] H. A. Kramers, Nature London 117, 775 (1926); R. d. L. Kronig, J. Opt. Soc. Am. 12, 547 (1926).

[24] E. Tuncer, N. Bowler, I. J. Youngs and K. P. Lymer Phil. Mag. 86, 2359 (2006).

[25] E. Tuncer Phys Rev B 71, 012101 (2005); E. Tuncer, J. Phys. D: Appl. Phys. 38, 223 (2005); E. Tuncer, Materials 3, 585 (2010); E. Tuncer, N. Bowler and I. J. Youngs, Physica B 373, 306 (2006); E. Tuncer and G. A. Niklasson, Optics Comm. 281, 4374 (2008); E. Tuncer, J. Phys.: Condens. Matter 17, L125 (2005); E. Tuncer, Phil. Mag. Lett., 85, 269 (2005).

[26] J. Ravier, F. Houze, F. Carmona, O. Schneegans, and H. Saadaoui, Carbon 39, 314; (2001); F. Houze, R. Meyer, O. Schneegans, and L. Boyer L, Appl. Phys. Lett. 69, 1975 (1996); F. Carmona, and J. Ravier, Carbon 40, 151 (2002). 


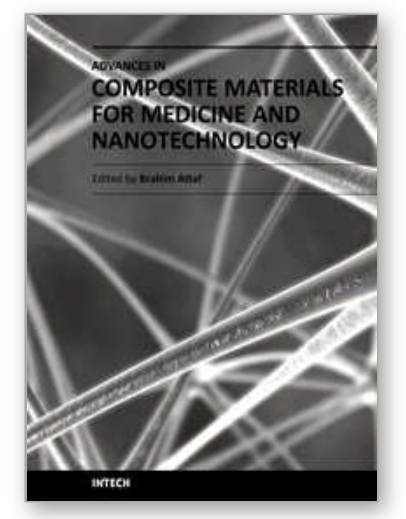

\section{Advances in Composite Materials for Medicine and Nanotechnology}

Edited by Dr. Brahim Attaf

ISBN 978-953-307-235-7

Hard cover, 648 pages

Publisher InTech

Published online 01, April, 2011

Published in print edition April, 2011

Due to their good mechanical characteristics in terms of stiffness and strength coupled with mass-saving advantage and other attractive physico-chemical properties, composite materials are successfully used in medicine and nanotechnology fields. To this end, the chapters composing the book have been divided into the following sections: medicine, dental and pharmaceutical applications; nanocomposites for energy efficiency; characterization and fabrication, all of which provide an invaluable overview of this fascinating subject area. The book presents, in addition, some studies carried out in orthopedic and stomatological applications and others aiming to design and produce new devices using the latest advances in nanotechnology. This wide variety of theoretical, numerical and experimental results can help specialists involved in these disciplines to enhance competitiveness and innovation.

\section{How to reference}

In order to correctly reference this scholarly work, feel free to copy and paste the following:

E. Tuncer, J. Belattar, M. E. Achour and C. Brosseau (2011). Broadband Spectral Analysis of Non-Debye Dielectric Relaxation in Percolating Heterostructures, Advances in Composite Materials for Medicine and Nanotechnology, Dr. Brahim Attaf (Ed.), ISBN: 978-953-307-235-7, InTech, Available from: http://www.intechopen.com/books/advances-in-composite-materials-for-medicine-andnanotechnology/broadband-spectral-analysis-of-non-debye-dielectric-relaxation-in-percolatingheterostructures

\section{INTECH}

open science | open minds

\section{InTech Europe}

University Campus STeP Ri

Slavka Krautzeka 83/A

51000 Rijeka, Croatia

Phone: +385 (51) 770447

Fax: +385 (51) 686166

www.intechopen.com

\section{InTech China}

Unit 405, Office Block, Hotel Equatorial Shanghai

No.65, Yan An Road (West), Shanghai, 200040, China

中国上海市延安西路65号上海国际贵都大饭店办公楼 405 单元

Phone: +86-21-62489820

Fax: $+86-21-62489821$ 
(C) 2011 The Author(s). Licensee IntechOpen. This chapter is distributed under the terms of the Creative Commons Attribution-NonCommercialShareAlike-3.0 License, which permits use, distribution and reproduction for non-commercial purposes, provided the original is properly cited and derivative works building on this content are distributed under the same license. 